\title{
A two-fluid model of nuclear rotations and surface vibrations
}

\author{
M A Z Habeeb†, R F Bishopł, J M Irvineł and M R Strayer\& \\ $\uparrow$ Department of Theoretical Physics, The University of Manchester, Manchester \\ M139PL, UK \\ \$Department of Theoretical Physics, The University of Manchester, Manchester \\ M139PL, UK and Daresbury Laboratory, Science Research Council, Warrington \\ WA4 4AD, UK \\ $\S$ Daresbury Laboratory, Science Research Council, Warrington WA44AD, UK
}

Received 7 November 1977

\begin{abstract}
A simple phenomenological two-fiuid model of nuclear collective motion previously used by the authors to discuss rotations is extended to include surface vibrations.

The model is applied to a study of the rare-earth nuclei ${ }^{154} \mathrm{Gd},{ }^{164} \mathrm{Er}$ and ${ }^{168} \mathrm{Yb}$ in an investigation of the rotation-vibration coupling in the presence of Coriolis antipairing effects and of the effects of Coriolis antipairing in the $\beta$ bands.
\end{abstract}

\section{Introduction}

In an earlier paper (Habeeb et al 1976, to be referred to as I) we have developed a simple two-fluid (namely normal and superfluid) model of nuclear rotations based upon the generalised linear flow of Cusson (1968). In this model the excitation energy of the member of the ground-state rotational band (GSRB) with angular momentum $J$ is given by

$$
\begin{aligned}
E_{J}\left(v_{\mathrm{n}}, v_{\mathrm{p}}, T\right)= & \frac{J(J+1) \hbar^{2}}{2 \mathscr{I}_{0} P} \frac{\left(1+\eta^{2}\right) T^{4}}{\left(1+\eta^{2} T^{6}\right)} \frac{A A_{\mathrm{n}} A_{\mathrm{p}}}{N F_{\mathrm{n}} A_{\mathrm{p}}+Z F_{\mathrm{p}} A_{\mathrm{n}}} \\
& +\frac{9}{128} k a_{0}^{2} \frac{\left(1-T^{2}\right)^{2}}{T^{4}}+\frac{1}{2} \sum_{\tau=\mathrm{n}, \mathrm{p}} C_{\tau}\left(v_{\tau}-\Delta_{\tau}\right)^{2}
\end{aligned}
$$

for a nucleus containing $N$ neutrons and $Z$ protons, and where the three terms represent respectively the rotational kinetic energy of the two-fluid drop, the centrifugal stretching (surface tension) energy, and the pairing energy with respect to the ground state. Here

$$
F_{\mathrm{\tau}}=\left[\xi \delta_{\tau}\left(1+\eta^{2} T^{6}\right)+\left(1-\eta^{2} T^{6}\right) v_{\tau}\right]^{2}
$$

and

$$
A_{\tau}=\left[\left(1+\eta^{2} T^{6}\right)^{2} v_{\tau}^{2}+2 \xi \delta_{\tau} v_{\tau}\left(1-\eta^{4} T^{12}\right)+\xi^{2} \delta_{\tau}^{2}\left(1+\eta^{2} T^{6}\right)^{2}\right]
$$

where the index $\tau$ labels either the protons $(\mathrm{p})$ or the neutrons $(\mathrm{n})$ which are considered to form two independent fluids of two components each. The nucleus is assumed 
to be incompressible, of uniform density $\rho$, and to occupy a spheroidal shape with semi-major and semi-minor axes $a$ and $b$ respectively. The ground-state values $a_{0}$ and $b_{0}$ and the ratio

$$
\eta=b_{0} / a_{0}
$$

are determined from the experimental ground-state quadrupole moments (Löbner et al 1970) assuming equivalent mass and charge distributions filling a volume $\frac{4}{3} \pi r_{0}^{3} A\left(A=N+Z, r_{0}=1.2 \mathrm{fm}\right)$. The ground-state rigid-body moment of inertia for such a nucleus is

$$
\mathscr{I}_{0}=\frac{4 \pi}{15} \rho a_{0}^{5} \eta^{2}\left(1+\eta^{2}\right)
$$

The ground-state neutron and proton pairing gaps $\Delta_{\tau}(\tau=n, p)$ are deduced from neighbouring odd-even mass differences. The pairing harmonic restoring strengths $C_{\text {t }}$ are taken from the work of $\mathrm{Ma}$ and Rasmussen $(1970,1974)$. With a Fermi gas assumption about the neutron and proton pairing correlation lengths we have

$$
\delta_{\tau}= \begin{cases}(N / Z)^{1 / 3} & \tau=\mathrm{n} \\ 1 & \tau=\mathrm{p} .\end{cases}
$$

The pairing gaps in the excited states $v_{\tau}$ and the centrifugal stretching parameter $T=b / b_{0}$ are determined variationally by minimising the energy expression $E_{J}\left(v_{\mathrm{n}}, v_{\mathrm{p}}, T\right)$ in (1) at each value of $J$. This leaves three adjustable parameters ( $P$, which measures the deviations of the nuclear rigid-body moment of inertia from the form given in equation (5), e.g. due to surface diffuseness etc, and which should be approximately unity, $k$, which measures the resistance to nuclear distortion, and $\xi$ which measures the pairing strength and which tends to infinity in the limit as the superfluid fraction tends to zero) to be fitted to the low-lying members of the GSRB. In I we showed that this model had the capability of reproducing the backbending phenomena frequently seen in rare-earth nuclei (Sorensen 1973, Stephens 1975). The appearance of backbending at some critical angular momentum was associated with a sudden sharp fall in the neutron pairing gap, i.e. a neutron Coriolis antipairing (CAP) effect.

In a refinement of the model (Habeeb et al 1977, to be referred to as II) we used an improved moment of inertia expression in place of $\mathscr{I}_{0}$ which allowed for surface diffuseness and set the parameter $P$ equal to unity. We also dropped the Fermi gas assumption which was used to relate the pairing correlations in the otherwise independent neutron and proton fluids, and combined $\xi$ and $\delta_{\tau}$ into new fitting parameters $\xi_{\tau}$. In this variation of the model the free parameters $k$ and $\xi_{\tau}$ were fitted to the three lowest members of the GSRB. With $\xi_{n}$ held constant for isotones and $\xi_{\mathrm{p}}$ held constant for isotopes we were able to reproduce the spectra of ${ }^{164,166} \mathrm{Er}$ and $166,168,170 \mathrm{Yb}$ which Faessler et al (1974) have suggested provide a good test of any model of nuclear rotations, since they display an interesting alternating pattern in their backbending behaviour.

It is well known, however, that backbending in the yrast band is sometimes associated with band crossing and that in other cases, even when the GSRB exhibits no backbending, the same phenomena may still occur in an excited band. This naturally leads us to consider an extension of our model which will allow for both rotations and shape vibrations. 


\section{The model}

\subsection{The classical kinetic energy}

We retain in this work the assumption introduced by Cusson and developed by us in I of a nuclear fluid flow which is linear in the particle coordinates. Consider the transformation matrix $\boldsymbol{M}(t)$ such that

$$
\dot{r}_{n}(t)=\mathbf{M}(t) \cdot r_{0 n}
$$

where $r_{n}(t)$ is the position vector of the $n$th particle at time $t$ in a space-fixed reference frame and $r_{0}$ is the position vector of the $n$th particle in a coordinate system in which all the particles are at rest. Then

$$
\dot{r}_{n}(t)=\chi(t) \cdot r_{n}(t)
$$

where

$$
\chi(t)=\dot{M} \cdot M^{-1} \equiv-\left(M \cdot \dot{M}^{-1}\right) .
$$

Equation (8) represents a differential constraint on the coordinates $\boldsymbol{r}_{n}(t)$.

Following Cusson we decompose $\boldsymbol{M}(t)$ in the form

$$
\boldsymbol{M}(t)=\boldsymbol{R}_{1}\left(\left\{\theta^{(1)}\right\}\right) \cdot \boldsymbol{S}(s) \cdot \tilde{\boldsymbol{R}_{2}}\left(\left\{\theta^{(2)}\right\}\right)
$$

where $\boldsymbol{R}_{1}$ and $\boldsymbol{R}_{2}$ are two orthogonal matrices defined by the two independent sets of Euler angles $\left\{\theta^{(1)}\right\}$ and $\left\{\theta^{(2)}\right\}$ respectively. $\boldsymbol{R}_{2}$ represents rotations of the nuclear fluid relative to a body-fixed coordinate frame and $\boldsymbol{R}_{1}$ represents rotations of this body-fixed frame relative to our space-fixed frame. $S(s)$ represents an irrotational shape change transformation, i.e. a pure scaling of position vectors. The assumption of nuclear incompressibility is contained in the requirement

$$
\operatorname{det} S=1 \text {. }
$$

In the pure rotation case $S$ is time independent:

$$
S(s)=S_{0}\left(s_{0}\right)
$$

To allow for shape vibrations we now allow $S$ to have a time-dependent part $S_{1}$ :

$$
S(s)=S_{0}\left(s_{0}\right) \cdot S_{1}(\delta s(t)) .
$$

Equation (9) can now be written

$$
\chi=\boldsymbol{R}_{1} \cdot \chi^{\prime} \cdot \tilde{\boldsymbol{R}}_{1}
$$

where

$$
\chi^{\prime}=\tilde{\boldsymbol{R}}_{1} \cdot \dot{\boldsymbol{R}}_{1}+\dot{S} \cdot S^{-1}+S \cdot \hat{\tilde{R}}_{2} \cdot R_{2} \cdot R_{2} \cdot S^{-1} .
$$

For the case of pure rotations we have

$$
\boldsymbol{\chi}_{\mathrm{rot}}^{\prime}=\tilde{\boldsymbol{R}}_{1} \cdot \dot{\boldsymbol{R}}_{1}+S_{0} \cdot \tilde{\boldsymbol{R}}_{2} \cdot \boldsymbol{R}_{2} \cdot \boldsymbol{S}_{0}^{-1}
$$

which was used as the basis for I. From equations (15) and (16) we define a vibrational component of $\chi^{\prime}$ by

$$
\chi_{\mathrm{vib}}^{\prime}=\chi^{\prime}-\chi_{\mathrm{rot}}^{\prime}
$$

We now write the vector $s$ which defines the surface of the nucleus in polar 
coordinates $(\sigma, \gamma)$, and the elements of the matrices in (15) and (16) are then all as given by Cusson except for the new matrix $\dot{S} . S^{-1}$ which is diagonal and has elements

$$
\left(\dot{S} \cdot S^{-1}\right)_{k l}=\delta_{k l} \sqrt{\frac{4}{3}} \dot{\sigma} \cos \left(\gamma-\frac{2}{3} k \pi\right) \quad k=1,2,3
$$

where we have assumed for simplicity that we only have axially symmetric vibrations, i.e. $\dot{\gamma}=0$.

The classical collective kinetic energy for a system of $N$ particles each of mass $m$ is

$$
T=\frac{1}{2} \sum_{n=1}^{N} m\left|\dot{r}_{n}(t)\right|^{2} .
$$

Substituting equations (8) and (14) into equation (19) we find that the total collective kinetic energy can be decomposed according to equation (17) into a rotational and a vibrational contribution:

$$
T=T_{\text {rot }}+T_{\text {vib }}
$$

where

$$
T_{\mathrm{vib}}=\frac{1}{2} \mathscr{B}(\sigma, \gamma) \dot{\sigma}^{2}
$$

with the vibrational inertial parameter $\mathscr{B}$ given by

$$
\begin{aligned}
\mathscr{B}(\sigma, \gamma)=\frac{8 \pi}{45}[ & \exp \left(2 \tau_{1} . s\right)(\cos \gamma+\sqrt{3} \sin \gamma)^{2} \\
& \left.+\exp \left(2 \tau_{2} . s\right)(\cos \gamma-\sqrt{3} \sin \gamma)^{2}+4 \exp \left(2 \tau_{3} . s\right) \cos ^{2} \gamma\right] \mathscr{B}^{\mathrm{Bohr}} .
\end{aligned}
$$

In terms of our polar coordinates

$$
\tau_{k} . s=\sqrt{\frac{4}{3}} \sigma \cos \left(\gamma-\frac{2}{3} k \pi\right)
$$

and the Bohr vibrational intertial parameter is

$$
\mathscr{B}^{\mathrm{Bohr}}=\frac{1}{2} \rho R_{0}^{5} .
$$

The rotational kinetic energy is

$$
T_{\text {rot }}=\frac{1}{2} \sum_{k=1}^{3}\left(\sum_{\tau=\mathrm{n}, \mathrm{p}} \mathscr{F}_{k}^{\mathrm{eff}}\left(\hat{\lambda}_{\tau},\left|B_{k}\right|\right)\right) \omega_{k}^{2}
$$

where $\omega_{1}, \omega_{2}$ and $\omega_{3}$ are the angular velocities about the $x, y$ and $z$ axes of the bodyfixed frame, and the corresponding effective moments of inertia are

$$
\mathscr{I}_{k}^{\mathrm{eff}}\left(\lambda_{\tau},\left|B_{k}\right|\right)=\frac{\left(\lambda_{\tau}+\mid B_{k}\right)^{2}}{\left(1+\lambda_{\tau}\left|B_{k}\right|+\lambda_{\tau}^{2}\right)} I_{0 k}
$$

which depend on the shape of the nucleus through the parameter

$$
\left|B_{k}\right|=\tanh \left(\beta_{k} \cdot s\right)
$$

with

$$
I_{0 k}=2 G_{0} \exp \left(-\tau_{k} . s\right) \cosh \left(\boldsymbol{\beta}_{k} . s\right)
$$

and where, in our polar coordinates,

$$
\beta_{k} \cdot s=2 \sigma \sin \left(\gamma-\frac{2}{3} k \pi\right)
$$


and $G_{0}$ is half the rigid-body moment of inertia of the equivalent sphere of radius $R_{0}$ :

$$
G_{0}=\frac{1}{5} M R_{0}^{2}=\frac{8}{15} \pi \mathscr{B}^{\text {Bohr }} .
$$

The parameters $\mathscr{I}_{k}^{\text {eff }}$ also depend on the pairing gaps $v_{\tau}$ through $\hat{\lambda}_{\tau}$, where

$$
\lambda_{\tau}=\xi \sqrt{\epsilon_{\mathrm{F}}^{\tau}} / v_{\tau}
$$

as in I.

As $v_{\tau}$ varies between the limits 0 and $\propto$ the effective moment of inertia varies between its rigid-body value and its irrotational flow value. However, the vibrational inertial parameter does not vary and always assumes its irrotational value. This result may at first sight appear strange since one may expect that as the system becomes rigid it cannot vibrate and this should be reflected in $\mathscr{B}(\sigma, \gamma)$. However, whether the system can vibrate or not is dictated not only by the inertial parameter but also by the strengths of the restoring forces, i.e. surface tension etc. This feature, that $\mathscr{B}(\sigma, \gamma)$ always takes its irrotational value, is a direct consequence of the linear flow assumption and the same result has recently been obtained by Gulshani and Rowe (1976) who find, in trying to generalise the Cusson treatment using group-theoretic techniques, that the vibrational inertia parameter for the general linear incompressible flow always assumes the irrotational flow value of Bohr and Mottelson (1969) for small deformations or the irrotational functional form given by Pal (1971) for a more exact treatment of ellipsoidal shapes corresponding to our approach.

\subsection{The quantal Hamiltonian}

The total Hamiltonian is assumed to consist of the kinetic energy terms discussed in the previous section plus some potential energy $V$ :

$$
H_{\text {total }}=T_{\text {rol }}+T_{\text {vib }}+V \text {. }
$$

We shall work in a representation in which $V$ is a $c$ number and we need only quantise the kinetic energy terms. We shall adopt the standard Pauli prescription according to which, if the Hamiltonian is expressible in the form

$$
H=\frac{1}{2} \sum_{\mu v} g_{\mu v}(\{q\}) \dot{q}_{\mu} \dot{q}_{v} \equiv \frac{1}{2} \sum_{\mu v} g^{\mu v} p_{\mu} p_{v}
$$

where the parameters $q_{\mu}$ are generalised coordinates with corresponding canonical momenta $p_{\mu}$ and $g^{\mu v}$ is the inverse of the matrix $g_{\mu v}$, then the quantal counterpart of $H$ is

$$
\hat{H}=-\frac{\hbar^{2}}{2} \sum_{\mu \nu} \frac{1}{|g|^{1 / 2}} \frac{\partial}{\partial q_{\mu \nu}}|g|^{1 / 2} g^{\mu \nu}(q) \frac{\partial}{\partial q_{\nu}} .
$$

Our expression for the kinetic energy, equation (20), is not yet in the form (33). However it can be cast into this form if we choose as our generalised coordinates

$$
\left(q_{1}, q_{2}, q_{3}, q_{4}\right) \equiv\left(\theta_{k}^{(1)}, k=1,2,3, \sigma\right)
$$

and using the definition

we have

$$
p_{\mu}=\partial H / \partial \dot{q}_{\mu}
$$

$$
p_{k}=j_{k}^{(1)}=\frac{\partial H}{\partial \dot{\theta}_{k}^{(1)}} \quad k=1,2,3
$$


which are the Euler generators of the collective rotation $\boldsymbol{R}_{1}$, and

$$
p_{4}=\mathscr{B}(\sigma, \gamma) \dot{\sigma} \text {. }
$$

The angular velocities $\omega_{1}, \omega_{2}$ and $\omega_{3}$ are given in terms of the Euler angles $\theta_{k}$ by

$$
\begin{aligned}
& \omega_{1}=\dot{\theta}_{2} \sin \theta_{3}-\dot{\theta}_{1} \sin \theta_{2} \cos \theta_{3} \\
& \omega_{2}=\dot{\theta}_{2} \cos \theta_{3}+\dot{\theta}_{1} \sin \theta_{2} \sin \theta_{3} \\
& \omega_{3}=\dot{\theta}_{1} \cos \theta_{2}+\dot{\theta}_{3}
\end{aligned}
$$

and hence equation (20) may be rewritten in the form of equation (33) with

$$
\begin{aligned}
g_{\mu v} & =\left[\begin{array}{llll}
g_{11} & g_{12} & g_{13} & 0 \\
g_{21} & g_{22} & 0 & 0 \\
g_{31} & 0 & g_{33} & 0 \\
0 & 0 & 0 & g_{44}
\end{array}\right] \\
g_{11} & =\mathscr{I}_{1} \sin ^{2} \theta_{2} \cos ^{2} \theta_{3}+\mathscr{I}_{2} \sin ^{2} \theta_{2} \sin ^{2} \theta_{3}+\mathscr{I}_{3} \cos ^{2} \theta_{2} \\
g_{12} & =g_{21}=\left(\mathscr{I}_{2}-\mathscr{I}_{1}\right) \sin \theta_{2} \sin \theta_{3} \cos \theta_{3} \\
g_{13} & =g_{31}=\mathscr{I}_{3} \cos \theta_{2} \\
g_{22} & =\mathscr{I}_{1} \sin ^{2} \theta_{3}+\mathscr{I}_{2} \cos ^{2} \theta_{3} \\
g_{33} & =\mathscr{I}_{3} \\
g_{44} & =\mathscr{B}
\end{aligned}
$$

and we have defined

$$
\mathscr{I}_{k}=\sum_{\tau} \mathscr{I}_{k}^{\mathrm{eff}}\left(\lambda_{\tau},\left|B_{k}\right|\right)
$$

Thus the quantal kinetic energy is simply

$$
\begin{aligned}
& \hat{T}=\hat{T}_{\text {rot }}+\hat{T}_{\text {vib }} \\
& \hat{T}_{\text {vib }}=-\frac{1}{2} \hbar^{2}\left[\mathscr{B}(\sigma, \gamma) \mathscr{I}_{1} \mathscr{I}_{2} \mathscr{I}_{3}\right]^{-1 / 2} \frac{\partial}{\partial \sigma}\left[\left(\frac{\mathscr{I}_{1} \mathscr{I}_{2} \mathscr{I}_{3}}{\mathscr{B}(\sigma, \gamma)}\right)^{1 / 2} \frac{\partial}{\partial \sigma}\right]
\end{aligned}
$$

and where $\hat{T}_{\text {rot }}$ is the usual Hamiltonian for the asymmetric top. It is easily shown that for small quadrupole deformations in the irrotational limit $v_{\tau} \rightarrow \infty, \hat{T}$ of equation (42) reduces to the Davydov-Chaban (1960) rotation plus axially symmetric vibration Hamiltonian.

The potential energy $V$, as in $\mathrm{I}$, is assumed to contain a centrifugal stretching term

$$
V_{\text {cent }}(\sigma)=\frac{1}{2} k\left(\frac{9}{64}\right) a_{0}^{2}\left(\exp \left[2\left(\sigma-\sigma_{0}\right) / \sqrt{3}\right]-1\right)^{2}
$$

where $\sigma_{0}$ is the ground-state value of $\sigma$, and a pairing term

$$
V_{\text {pair }}=\frac{1}{2} \sum_{\tau=n, p} C_{\tau}\left(v_{\tau}-\Delta_{\tau}\right)^{2}
$$

which is independent of $\sigma$. 


\subsection{The Schrödinger equation}

Having defined the quantal Hamiltonian in the previous section we now look for solutions to the Schrödinger equation

$$
\hat{H} \Psi=(\hat{T}+V) \Psi=E \Psi
$$

subject to suitable boundary conditions. Since $V_{\text {pair }}$ is independent of $\sigma$ the boundary conditions are independent of $V_{\text {pair }}$. The general boundary condition is that $\Psi$ should be single valued. The general solution of (45) may be expanded in the form $\Psi_{J M}^{x}\left(\sigma, \gamma,\left\{\theta^{(1)}\right\}\right)$

$$
=\sum_{K} g_{K}^{\alpha J}(\sigma, \gamma)\left(\frac{2 J+1}{16 \pi^{2}\left(1+\delta_{K 0}\right)}\right)^{1 / 2}\left(\mathscr{D}_{M K}^{J}\left(\left\{\theta^{(1)}\right\}\right)+(-1)^{J} \mathscr{D}_{M-K}^{J}\left(\left\{\theta^{(1)}\right\}\right)\right) .
$$

Here $J$ is the total angular momentum, $M$ is its projection on the third axis of the space-fixed frame and $K$ is its projection on the third axis of the body-fixed frame, and $\alpha$ represents any other quantum numbers. Substitution of (46) into (45) leads to the second-order differential equation for the vibrational functions $g_{K}^{\alpha J}(\sigma, \gamma)$

$$
\begin{aligned}
\hat{T}_{\mathrm{vib}} g_{K}^{\alpha J}+g_{K-2}^{\alpha J} & {\left[\frac{1}{4}\left(\frac{\hbar^{2}}{2 \mathscr{I}_{1}}-\frac{\hbar^{2}}{2 \mathscr{I}_{2}}\right)[(J+K)(J+K-1)(J-K+1)(J-K+2)]^{1 / 2}\right] } \\
+ & g_{K+2}^{\alpha J}\left[\frac{1}{4}\left(\frac{\hbar^{2}}{2 \mathscr{I}_{1}}-\frac{\hbar^{2}}{2 \mathscr{I}_{2}}\right)[(J-K)(J-K-1)(J+K+1)(J+K+2)]^{1 / 2}\right] \\
+ & g_{K}^{\alpha J} f_{J}^{K}=\epsilon_{J}^{\alpha K} g_{K}^{\alpha J}
\end{aligned}
$$

where

$$
f_{J}^{K}=\frac{1}{2}\left(\frac{\hbar^{2}}{2 \mathscr{I}_{1}}+\frac{\hbar^{2}}{2 \mathscr{I}_{2}}\right)\left[J(J+1)-K^{2}\right]+\frac{\hbar^{2} K^{2}}{2 \mathscr{I}_{3}}+V_{\mathrm{cent}} .
$$

For simplicity we shall now restrict ourselves to axially symmetric nuclear shapes, i.e. $\gamma=0$, which in turn implies $\mathscr{I}_{2}=\mathscr{I}_{1}, K=0$ and $J$ restricted to $0,2,4 \ldots$ Equation (47) then reduces to

$$
\left(Q(\sigma) \hat{M}+f_{J}(\sigma)\right) g_{0}^{\alpha J}(\sigma)=\epsilon_{J}^{\alpha} g_{0}^{\alpha J}(\sigma)
$$

with

$$
\begin{aligned}
& Q(\sigma)=-h^{2} /\left[2\left(\mathscr{B} \mathscr{I}_{1}^{2} \mathscr{I}_{3}\right)^{1 / 2}\right] \\
& \hat{M}=A(\sigma) \partial^{2} / \partial \sigma^{2}+(\partial A(\sigma) / \partial \sigma) \partial / \partial \sigma \\
& A(\sigma)=\left(\mathscr{I}_{1}^{2} \mathscr{I}_{3} / \mathscr{B}\right)^{1 / 2} \\
& f_{J}(\sigma)=\hbar^{2} J(J+1) / 2 \mathscr{I}_{1}+V_{\text {cent }}(\sigma)
\end{aligned}
$$

which is solved subject to the natural boundary conditions

$$
g_{0}^{\alpha J}(0)=g_{0}^{\alpha J}(\infty)=0 .
$$

The total energy of the nucleus is then obtained by adding the pairing potential to the eigenvalues $\epsilon_{J}^{\alpha}$ :

$$
E_{J}^{\alpha}\left(v_{\mathrm{n}}, v_{\mathrm{p}}\right)=\epsilon_{J}^{\alpha}\left(v_{\mathrm{n}}, v_{\mathrm{p}}\right)+\frac{1}{2} \sum_{\tau=\mathrm{n}, \mathrm{p}} C_{\tau}\left(v_{\tau}-\Delta_{\tau}\right)^{2}
$$


where we note that the $\epsilon_{J}^{x}$ are functions of $v_{\tau}$ through their dependence on the effective moments of inertia.

We shall now identify the lowest two eigenvalues for each value of $J$ with the GSRB and the $\beta$-vibrational band. Note that with the restrictions we have imposed, in the limit of small oscillations we have

$$
\sigma \rightarrow \sqrt{\frac{15}{16 \pi}} \beta
$$

where $\beta$ is the Bohr-Mottelson (1969) vibrational parameter.

As in $\mathrm{I}, v_{\mathrm{n}}, v_{\mathrm{p}}$ and $T$ are treated as variational parameters while $k$ (equation (43)) and $\xi$ (equation (31)) are fitting parameters. The ground-state deformations are taken from measured quadrupole moments and the pairing parameters are taken from odd-even mass differences and the work of Ma and Rusmussen $(1970,1974)$. Again we introduce a parameter $P$ multiplying the effective moment of inertia $\mathscr{I}_{3}$ which is meant to take into account uncertainties due to our assumption of a uniform density. As in $\mathrm{I}$, we argue that $P$ is close to unity and the need for it could be removed if we employed the refinements discussed in II.

\section{Model calculations}

To indicate the type of results that can be obtained with this model, we have carried out a select number of calculations for illustrative purposes. In these calculations

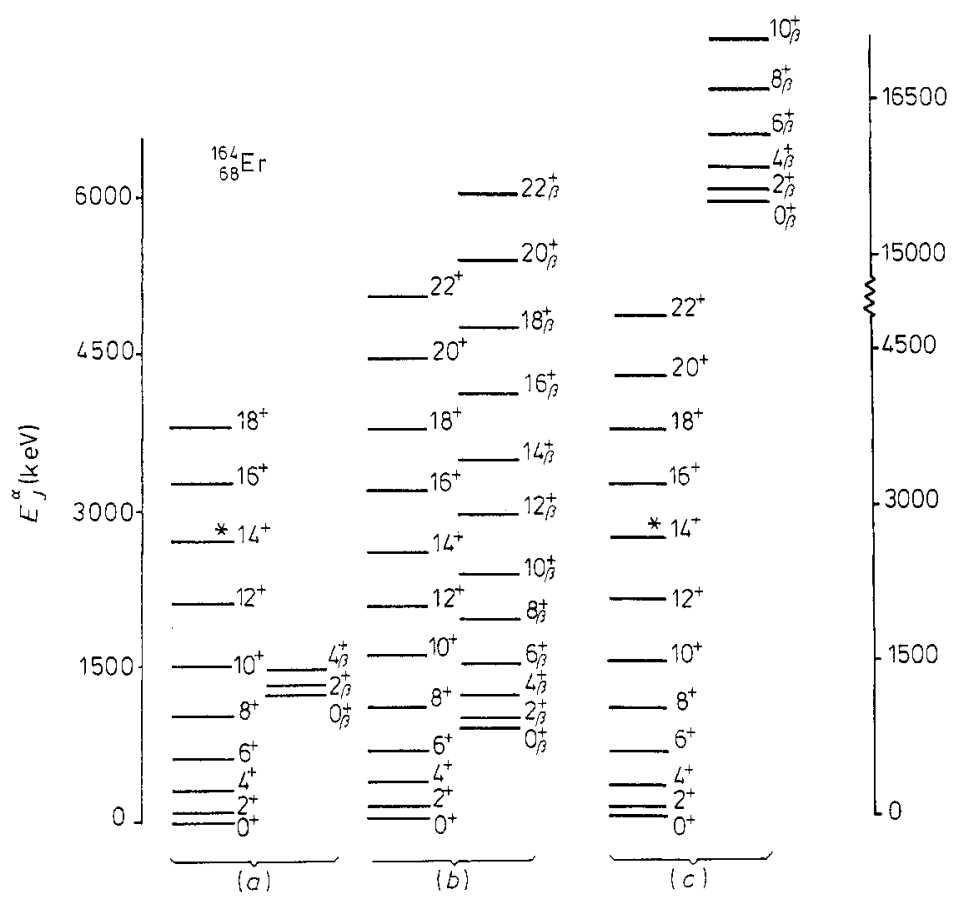

Figure 1. The spectra of ${ }_{68}^{164} \mathrm{Er}$ : $(a)$ experimental, $(b)$ with $P=1.0$ and $(c)$ with $P=1.6$. In both calculated spectra $(b)$ and $(c)$ the parameters $k$ and $\xi$ were chosen to give a best $\chi^{2}$ fit to the ground-state rotational band. An asterisk indicates backbending at this member of the rotational band. For each spectrum, the levels on the left-hand side are for the GSRB, those on the right-hand side are for the $\beta$-vibrational band. 


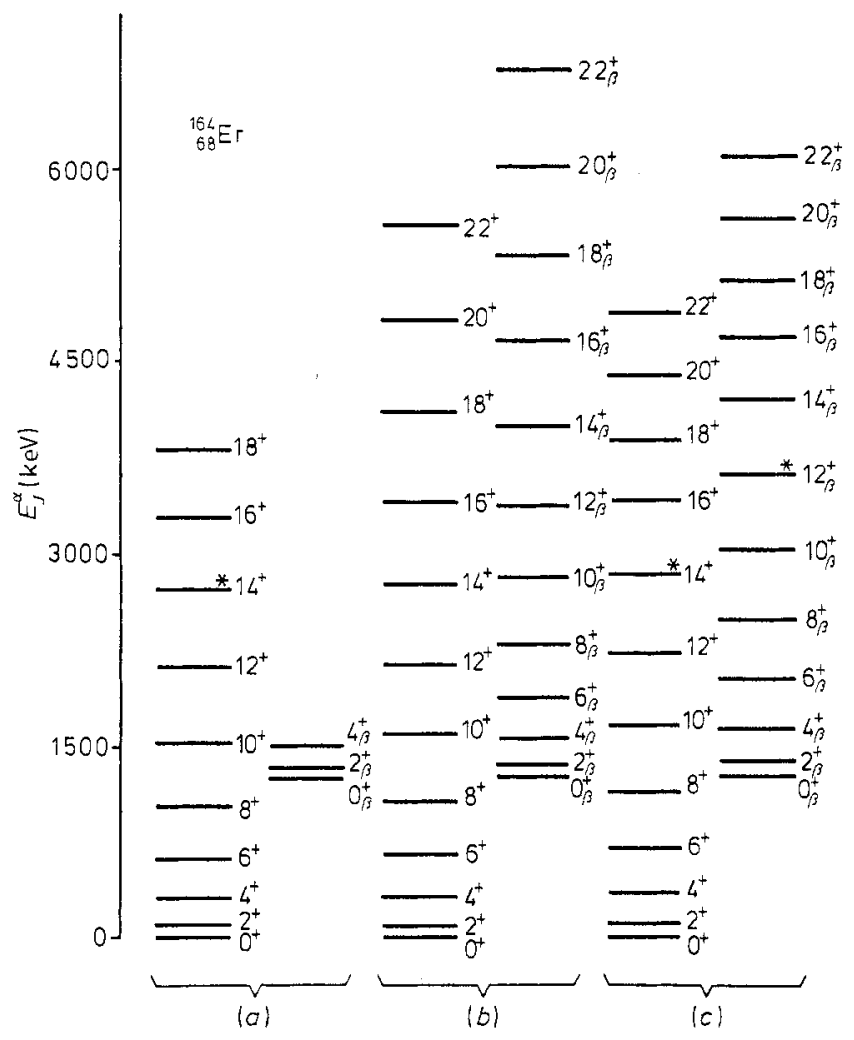

Figure 2. As figure 1 but with $k$ chosen to reproduce the $\beta$-band $0^{+}$state and $\xi$ chosen to give a best fit to the ground-state band.

we have chosen ${ }^{164} \mathrm{Er}$ as an example of a nucleus known to backbend in its GSRB, ${ }^{168} \mathrm{Yb}$ as an example of a nucleus known not to backbend, at least as far as the known members of the GSRB are concerned, and ${ }^{154} \mathrm{Gd}$ as an example of a nucleus known to exhibit band crossing.

In figure 1 we compare our calculated spectra for ${ }_{68}^{164} \mathrm{Er}$ with experiment. All experimental data are taken from Sakai (1975). Here, as in I, $k$ and $\xi$ are chosen to give the best $\chi^{2}$ fit to the GSRB and we have exhibited our results for $P=1.0$ and $P=1 \cdot 6$, the latter value being the one which gave us a best fit to the critical GSRB angular momentum in I. We see that with $P=1.0$ the $\beta$-vibration band-head is reasonably well represented but as in I the GSRB does not exhibit backbending up to the $22^{+}$member. On the other hand, with $P=1.6$ we reproduce excellently the GSRB including backbending at the $14^{+}$member but now the $\beta$-vibrational bandhead is very badly given. Since $k$ measures the resistance to stretching of the nuclear drop, we might expect the $\beta$-vibrational band-head to be extremely sensitive to this parameter. In figure 2 we present results for the case where $k$ is fixed to give the observed $\beta$ band-head and the single parameter $\xi$ is chosen to give a best fit to the GSRB. Now with $P=1.0$ there is a slight extension of the GSRB compared with experiment and still no backbending is predicted. However, with $P=1.6$ we are again able to give a perfect reproduction of the observed GSRB including the backbending at the $14^{+}$member and an excellent description of the observed members of the $\beta$ band. Our calculations predict a backbending in this band at the $12^{+}$ 

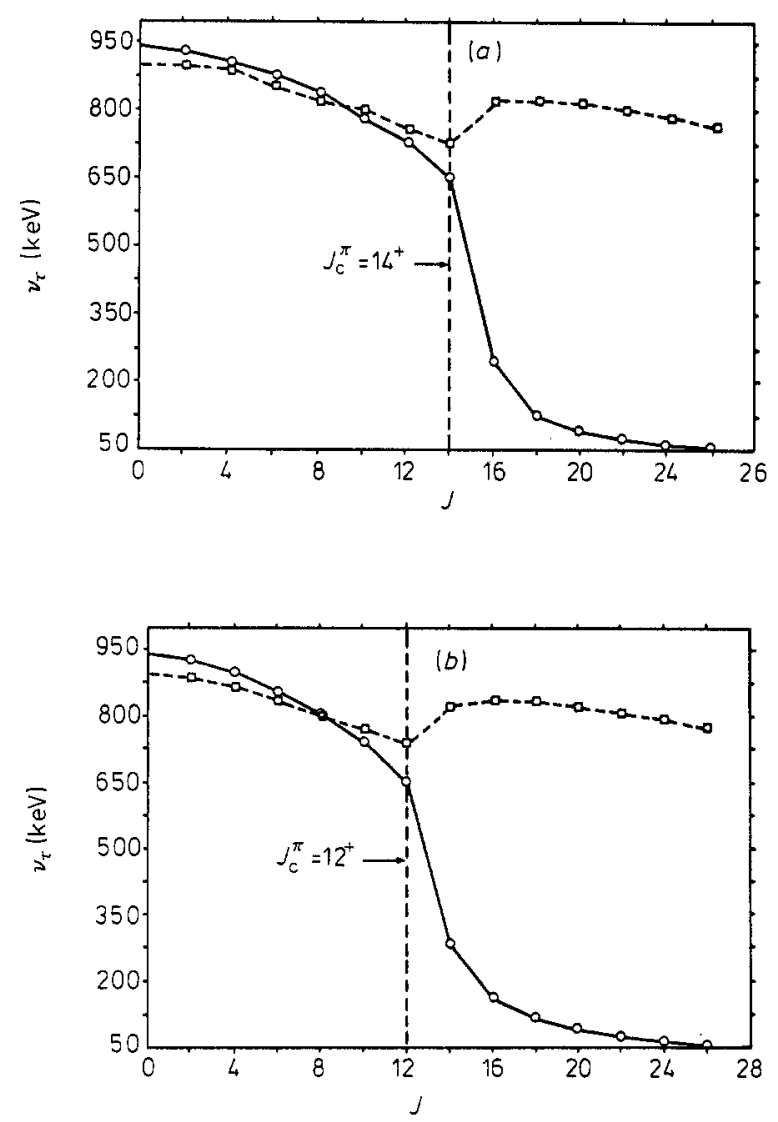

Figure 3. The neutron $(O)$ and proton $(\square)$ pairing gaps $v_{\tau}(\tau=n, p)$ calculated for ${ }_{68}^{164} \mathrm{Er}$ as a function of $J(a)$ for the ground-state band and $(b)$ for the $\beta$ band.

member. In figure 3 we present plots of our calculated pairing gap parameters $v_{\tau}$ and we see that in both the GSRB and the $\beta$ band the backbending is associated with a neutron CAP effect.

In figure 4 we compare our calculated spectra for ${ }_{70}^{168} \mathrm{Yb}$ with experiment, where again $k$ was chosen to reproduce the observed $\beta$ band-head and $\xi$ chosen to give a best fit to the GSRB. With $P=1.0$ there is no backbending in either the GSRB or the $\beta$ band and the GSRB is in good agreement with the observed experimental levels. At $P=1.6$ backbending is predicted at the $16^{+}$member of the GSRB and at the $14^{+}$member of the $\beta$ band. However, now the GSRB is seriously compressed compared with experiment. The best representation of the GSRB is obtained with $P=1.3$ (and see I) and now the predicted backbending is delayed to the $24^{+}$to $26^{+}$member of the GSRB and to $\sim 20^{+}$member of the $\beta$ band. In both cases the backbending is associated with neutron CAP.

Turning now to ${ }_{64}^{154} \mathrm{Gd}$ we once more fix $k$ to reproduce the observed $\beta$ band-head. There is now some ambiguity in fitting $\xi$ since it is known that in this nucleus there is a band crossing, and rather than refer to the bands as the GSRB and the $\beta$ band we should describe them as the yrast and the yrare bands. To avoid such ambiguity we now choose $\xi$ to give a best fit to the first four members of the GSRB (yrast band) and in figure 5 we compare our results with experiment. We first note, 

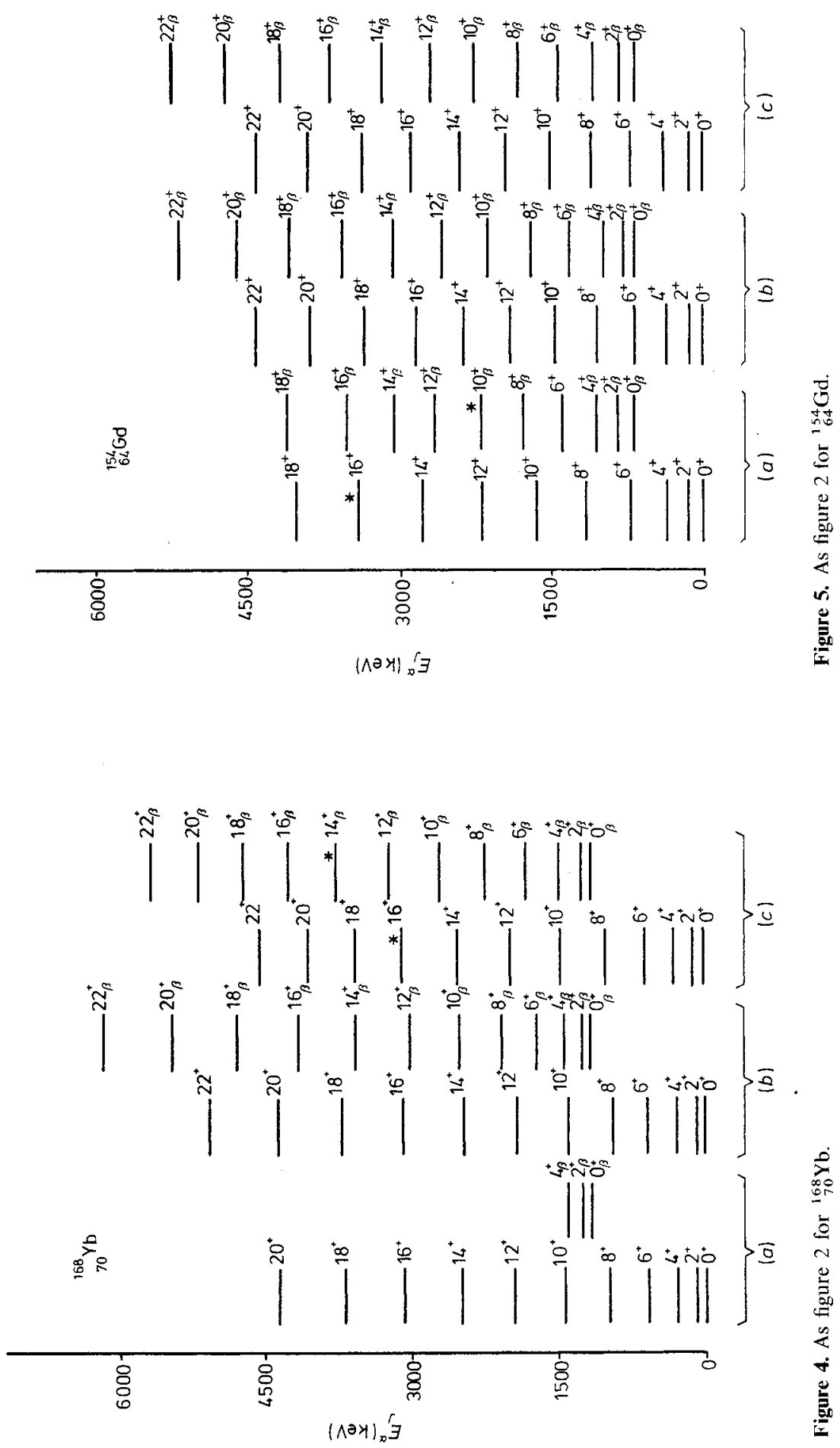


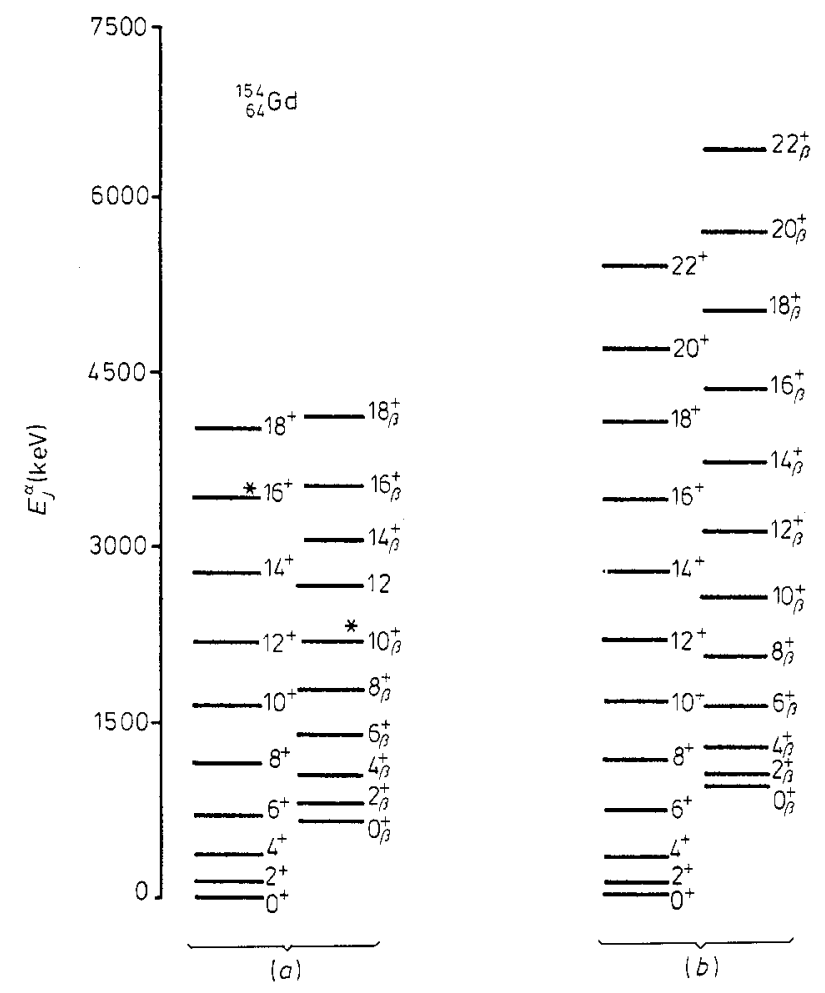

Figure 6. As figure 1 for ${ }_{64}^{154} \mathrm{Gd}$ with $P=1.0$.

as observed in I, that the results for this nucleus are extremely insensitive to the value of $P$. We note that we obtain an extremely good representation of the observed $\beta$ band without reproducing the backbending observed at the $10^{\circ}$ member (Khoo et al 1973). It should be noted, however, that the higher members of this band have not been confirmed experimentally. Our GSRB/yrast band is significantly compressed compared with experiment and this effectively prevents the band matching at $\sim 16^{+}$ which is observed. The agreement between experiment and our calculations on the GSRB can be improved significantly by fitting both $k$ and $\xi$ to give a best GSRB. The results are again very insensitive to the value of $P$ and are presented in figure 6 for $P=1.0$. We see that in this case we obtain a perfect match to the GSRB/yrast band up to the $18^{+}$level. The $\beta$ band-head is now off by $\sim 200 \mathrm{keV}$ and the $\beta$ band is significantly expanded compared with experiment and shows no sign of backbending at the $10^{+}$level or of band matching at the $16^{+}$level. The reasons for these effects await a more detailed study of this nucleus.

We have extended our model to allow for both rotations and surface vibrations. This allows for the possibility of describing the backbending effect either by Coriolis antipairing or band crossing. In ${ }_{68}^{164} \mathrm{Er}$ we find, as before, that we predict backbending at the observed $14^{+}$member of the ground-state band and also at the, as yet unobserved, $12^{+}$member of the $\beta$ band. In both cases the effect is predicted to be due to Coriolis antipairing. In ${ }_{70}^{168} \mathrm{Yb}$ we predict that no backbending should occur in either the ground-state band or the $\beta$ band below the $20^{+}$members, in agreement with current observations. In both these nuclei the detailed results are sensitive to the value of the parameter $P$ introduced to take account of our simplified assumption 
of a uniform nuclear density distribution. As discussed in II, when this assumption is dropped the parameter $P$ can be removed from our calculations without destroying the excellent description of these nuclei and their neighbouring isotopes.

In ${ }^{154} \mathrm{Gd}$ our results are insensitive to the value of $P$ and always fail to reproduce the observed backbending at the $16^{+}$member of the yrast band and the $10^{+}$member of the yrare band. Our model, of course, does not contain the possibility of rotational alignment and it may be, as suggested by Stephens (1975), that this is required for a description of this nucleus.

In conclusion we feel that the preliminary results for the model calculations presented here are sufficiently encouraging to warrant further study.

\section{Acknowledgment}

We are grateful to Professor R Y Cusson for private correspondence on particular points of this work.

\section{References}

Bohr A and Mottelson B R 1969 Nuclear Structure vol 1 (Mass: Benjamin)

Cusson R Y 1968 Nucl. Phys. A 114289

Davydov A S and Chaban A A 1960 Nucl. Phys. 20499

Faessler A, Grummer F, Lin L and Urbano J 1974 Phys. Lett. 48B 87

Gulshani P and Rowe D J 1976 Can. J. Phys. 54970

Habeeb M A Z, Bishop R F, Irvine J M and Strayer M R 1976 Nucl. Phys. A 272174

1977 J. Phys. G: Nucl. Phys. 3 L93

Khoo T L, Bernthal F M, Boyno J S and Warner R A 1973 Phys. Rev. Lett. 311146

Löbner K E G, Vetter M and Honig V 1970 Nuclear Data Tables 7495

Ma C W and Rasmussen J O 1970 Phys. Rev. C 2798

—1974 Phys. Rev. C 91083

Pal M K 1971 Trieste preprint IC/71/42

Sakai M 1975 Atomic Data and Nuclear Data Tables 15513

Sorensen R A 1973 Rev. Mod. Phys. 45353

Stephens F S 1975 Rev. Mod. Phys. 4743 